\title{
Correction to: Triggering of eryptosis, the suicidal erythrocyte death, by phenoxodiol
}

\author{
Madeline Fink $^{1}$ • Abdulla Al Mamun Bhuyan ${ }^{1}$ • Bernd Nürnberg ${ }^{1}$ • Caterina Faggio ${ }^{2}$ • Florian Lang ${ }^{3,4}$
}

Published online: 23 July 2019

(C) Springer-Verlag GmbH Germany, part of Springer Nature 2019

\section{Correction to: Naunyn-Schmiedeberg's Archives of Pharmacology https://doi.org/10.1007/s00210-019-01681-8}

The original version of this article contains several mistakes due to the below missed corrections:

Line 21: DELETE the "," after "scatter" and DELETE the "significantly increased"; ADD the "significantly increased" after "Fluo3 fluorescence and"

LIne 23: add "In conclusion, phenoxodiol" instead of Phenoxodiol; DELETE Ca2+ entry

Line 24: DELETE and

Line 155: decreased INSTEAD OF increased

Line 200: decreases INSTEAD OF increases
Line 201: and INSTEAD OF, but

Line 204: DELETE could still have partially been due to increases of $[\mathrm{Ca} 2+] \mathrm{i}$, but

Line 205: REMOVE this

Line 234: DELETE Ca2+ entry and

The original article has been corrected.

Publisher's note Springer Nature remains neutral with regard to jurisdictional claims in published maps and institutional affiliations.

The online version of the original article can be found at https://doi.org/ 10.1007/s00210-019-01681-8

Florian Lang

florian.lang@uni-tuebingen.de

1 Department of Phamacology and Experimental Therapy, Eberhard-Karls-University of Tuebingen, Tübingen, Germany

2 Department of Chemical, Biological, Pharmaceutical and Environmental Sciences, University of Messina, Viale Ferdinando Stagno d'Alcontres, S. Agata, Messina, Italy

3 Department of Internal Medicine III, Eberhard-Karls-University of Tuebingen, Tübingen, Germany

4 Department of Vegetative \& Clinical Physiology, University of Tübingen, 72074 Tübingen, Germany 